\title{
APPLICATION OF TIME SERIES ANALYSIS FOR BRIDGE MONITORING
}

\author{
Piotr Omenzetter (corresponding author) \\ Department of Civil and Environmental Engineering \\ The University of Auckland \\ Private Bag 92019 \\ Auckland \\ New Zealand \\ E-mail: p.omenzetter@auckland.ac.nz \\ Tel: $+64-9-3737599$ ext. 88138 \\ Fax: +64-9-3737462 \\ James Mark William Brownjohn \\ Department of Civil and Structural Engineering \\ The University of Sheffield \\ United Kingdom
}




\begin{abstract}
Despite the recent considerable advances in structural health monitoring (SHM) of civil infrastructure, converting large amount of data from SHM systems into usable information and knowledge remains a great challenge. This paper addresses the problem through analysis of time histories of static strain data recorded by an SHM system installed in a major bridge structure and operating continuously for a long time. The reported study formulates a vector seasonal autoregressive integrated moving average (ARIMA) model for the recorded strain signals. The coefficients of the ARIMA model are allowed to vary with time and are identified using an adaptive Kalman filter. The proposed method has been used for analysis of the signals recorded during construction and service life of the bridge. By observing various changes in the ARIMA model coefficients, unusual events as well as structural change or damage sustained by the structure can be revealed.
\end{abstract}




\section{INTRODUCTION}

The traditional assessment of the state of in-service civil infrastructure using periodic visual inspections and simple localized testing, such as the tap test, tends to produce subjective and inaccurate evaluations of structural safety and reliability (Phares et al., 2001). This is caused by the various shortcomings of visual inspection, such as high manpower demand, often insufficient frequency, inaccessibility of critical parts of the structure, and lack of information on actual loading. The resulting shortage of appropriate information pertaining to the structural health may result in incorrect decisions regarding infrastructure asset management, including maintenance, retrofitting, or replacement. For example, as a result of poor quality of structural health evaluation some structures may be retrofitted or replaced, while in fact they are still sound, or, on the other hand, existing damages in other structures may not be identified until they become expensive to repair or dangerous for structural integrity.

Motivated by the shortcomings of traditional inspection techniques on the one hand, and availability of affordable hardware and advances in analytical signal processing techniques on the other, engineers and researchers turned their attention to instrumented structural health monitoring (SHM) systems to complement and enhance visual inspection. Aktan et al. (2002) define SHM as a process involving tracking any aspect of structural performance or health by measuring data and interpreting them in conjunction with application specific knowledge so that structural condition and reliability can be quantified objectively. Nevertheless, despite vigorous research and considerable advances in SHM of civil infrastructure, notably bridges, common, efficient and reliable enhancement, let alone replacement, of visual inspection by instrumented monitoring systems is still some way into the future. In fact, it has become obvious that the current understanding of the complexity of materials, structures and their behaviour in actual environmental and operational conditions 
are far from being satisfactory from the point of view of efficient usage and understanding of measurements obtained via SHM.

One of the major challenges is to make sense of large amounts of data that continuously operating SHM systems produce. For the success of SHM, these data need to be reduced into manageable volumes and forms, and then information needs to be extracted and knowledge about structural condition created. There are likely to be major differences between the relatively basic expectations and requirements of infrastructure managers and the ambitions of systems designers, the latter usually originally academic. For example, bridge managers first of all wish to know if the structure is safe for continued operation, e.g. after and onerous event such as an earthquake or typhoon. So far, the greatest beneficiary from SHM has been the academic community who gained insight into behavior and performance of various exotic structures, such as long span bridges and high-rise buildings. Lower-profile but ultimately equally important and useful developments in SHM have been in optimal monitoring approaches for medium and short span bridges. There is a long history of research in full-scale testing for highway bridge assessment (Salane et al., 1981; Bakht and Jaeger, 1990). For smaller bridges, global response is more sensitive to defects, visual inspection is less frequent and SHM systems can make a real contribution (Alampalli and $\mathrm{Fu}, 1994$; Heywood et al., 2000).

The possibility of a SHM system being able to detect reliably damage that is not visually obvious has apparently not been achieved yet, and substantially more research is required. It is interesting to note that the majority of studies try to identify damage from dynamic modal characteristics, such as natural frequencies or mode shapes. While vibration data remain valuable, they need to be integrated with quasi-static response data as well as data from dynamic and quasi-static loading. Also, only a limited number of papers take into account the variability of SHM-identified dynamic parameters due to environmental and 
operational conditions, such as temperature, moisture, wind and others (Cornwell et al., 1999; Kullaa, 2002; Mahmoud et al., 2001; Wood, 1992). It was demonstrated (Farrar et al., 2000; Sohn et al., 1999) that if this variability is neglected, drawing reliable conclusions about the structural condition is very difficult. This is because changes in modal parameters due to environmental and operational factors may well exceed those caused by an even severe damage.

A novel, interesting approach for damage detection, which does not use the modal characteristics, was presented in Sohn et al. (2000) and Sohn et al. (2001). They modeled dynamic signals recorded on two different mechanical systems (a bridge pier and a patrol boat) at various damage states using autoregressive (AR) time series models. By statistically examining changes in AR model coefficients, they were able to classify signals as coming from either undamaged or damaged systems. It seems, however, that their original approach is more suitable for periodic or post-event monitoring, where a structure is respectively examined either at preset time intervals or after major onerous events such as an earthquake or typhoon, and measurement are compared to a baseline data set. In the case of continuous monitoring, it will be worthwhile to trace the system behavior permanently and detect changes as soon as possible after new data are available. The present study uses this approach and is devoted to modeling of time histories of static, hourly sampled strains recorded by an SHM system installed in a major bridge structure and operating continuously for a long time period. A vector seasonal autoregressive integrated moving average (ARIMA) model is established for the recorded strains. The coefficients of the ARIMA model are allowed to vary with time and are identified on-line using an adaptive Kalman filter. By observing various changes in the model coefficients, unusual events as well as structural change or damage sustained by the structure can be revealed. Such events or structural changes may result, among other causes, from a sudden settlement of foundation, ground movement, 
excessive traffic load or failure of post-tensioning cables. The proposed method has been applied to the strains recorded under normal operational and environmental conditions during construction of the structure and also when the bridge was in normal service.

\section{MONITORING OF THE BRIDGE}

The subject of the reported monitoring was the Singapore-Malaysia Second Link, also referred to as the Tuas Link (Fig. 1). This section of the paper is intended to give only the basic outline of the structure and the monitoring program, while more details can be found in Moyo and Brownjohn (2002a) and Omenzetter et al. (2004). The structure serves as a road crossing between the Island of Singapore and Malaysian Peninsula. The bridge, opened in 1997, is about $1.9 \mathrm{~km}$ long and comprises 27 spans. The main span of the Singapore's side is $92 \mathrm{~m}$ long. The bridge was cast in-situ using the balanced cantilever method, to enable the navigation channel to be kept free throughout the construction, and post-tensioned. The crosssection of the post-tensioned, continuous box girder varies in depth from $2.6 \mathrm{~m}$ to $6.5 \mathrm{~m}$ along the bridge length.

The instrumentation used for short-term and long-term monitoring of performance under environmental and traffic loads consists of four data loggers, twelve vibrating wire strain gauges, twelve pressure cells, forty four thermocouples and one tri-axial accelerometer. The sensors are distributed in three segments of the main span, namely Segment 23 (the middle of the span), Segment 27 (approximately one third of the span length), and Segment 31 (approximately one quarter of the span length). The locations of strain gauges in the cross section of the girder are shown in Figure 2. Three data loggers are responsible for static measurements, that is strains, stresses and temperature, and one data logger is responsible for dynamic measurements. All the data loggers are connected to a host computer, resident in the bridge, and remotely accessible via a modem. The monitoring program was divided into two 
components, namely static and dynamic monitoring. Static monitoring, which is the focus of this paper, involved the acquisition of stress, strain and temperature data from embedded sensors at hourly intervals.

\section{THEORY}

Continuously operating SHM systems generally produce various "raw" signals, such as displacements, accelerations, strains, stresses, temperatures, wind velocities, or signals resulting from some form of analytical processing of the raw data, e.g. natural frequencies or power spectra. Because of the character of signals recorded by SHM systems, i.e. time series sampled over long periods of time and at regular intervals, such data naturally lend themselves to examination using the extensive and proven tools offered by the time series analysis and statistical process control. The concepts of the time series analysis have successfully been applied to numerous problems, notably in the field of econometrics, where they have been used, for example, to investigate stock prices, production and prices of various commodities, and interests rates (Wei, 1993). Little has been reported, with the exception of the aforementioned study by Sohn et al. (2000), about application of time series analysis in the area of SHM of civil infrastructure. Another publication is that of Moyo and Brownjohn (2002b) who used intervention analysis for assessing the impact of various events during construction of a bridge on the recorded time series of strains. The present study uses several existing procedures of the time series analysis to understand and extract information from the strain data recorded on a bridge structure. The main objective is to identify abrupt events sustained by the bridge and possible structural change or damage. The procedure consists of the following steps and uses the following methods:

- The coefficients of a vector seasonal ARIMA model of the recorded strain signals are identified using an adaptive Kalman filter. 
- The coefficients of the ARIMA model are statistically examined using an outlier detection technique in order to find unduly large variations in their values.

- The effect of unusual variations of ARIMA coefficient values is classified as temporary or permanent and quantified using the intervention analysis.

\subsection{Vector seasonal ARIMA model for strains}

Observations of systems outputs recorded at certain time intervals are related to external inputs and often also to their past values. This correlation may extend over just a few proceeding observations, but can as well continue over long time periods. For example, measurements of air temperature at a given location will usually show significant correlation between temperatures recorded at daily and yearly intervals. To account for the various correlations between measurements, ARIMA models are often used.

Consider the following general form of a vector seasonal ARIMA model (Wei, 1993):

$$
\mathbf{D}(B) \mathbf{D}^{(S)}(B) \boldsymbol{\Phi}_{t}(B) \boldsymbol{\Phi}_{t}^{(S)}(B) \mathbf{x}_{t}=\boldsymbol{\Theta}_{t}(B) \boldsymbol{\Theta}_{t}^{(S)}(B) \mathbf{e}_{t}
$$

where $\left\{\mathbf{x}_{t}\right\}(t=1,2, \ldots, N)$ is the $p$-dimensional vector of analyzed signal's time series, and $\left\{\mathbf{e}_{t}\right\}$ is a zero mean multivariate Gaussian white noise. $B$ denotes the backshift operator which can be defined through its action on an arbitrary time series $\left\{\mathbf{v}_{t}\right\}$ in the following way:

$$
B \mathbf{v}_{t}=\mathbf{v}_{t-1}
$$

$\boldsymbol{\Phi}_{t}(B), \boldsymbol{\Phi}_{t}^{(S)}(B), \boldsymbol{\Theta}_{t}(B)$, and $\boldsymbol{\Theta}_{t}^{(S)}(B)$ are all matrix polynomials in the backshift operator:

$$
\begin{aligned}
\boldsymbol{\Phi}_{t}(B) & =\mathbf{I}-\sum_{i=1}^{r} \mathbf{A}_{t, i} B^{i} \\
\boldsymbol{\Phi}_{t}^{(S)}(B) & =\mathbf{I}-\sum_{i=1}^{R} \mathbf{A}_{t, i}^{(S)} B^{S i} \\
\boldsymbol{\Theta}_{t}(B) & =\mathbf{I}-\sum_{i=1}^{m} \mathbf{C}_{t, i} B^{i}
\end{aligned}
$$




$$
\mathbf{\Theta}_{t}^{(S)}(B)=\mathbf{I}-\sum_{i=1}^{M} \mathbf{C}_{t, i}^{(S)} B^{S i}
$$

where $\mathbf{A}_{t, i}, \mathbf{A}_{t, i}^{(S)}, \mathbf{C}_{t, i}$, and $\mathbf{C}_{t, i}^{(S)}$ are $p \times p$ matrices, and $\mathbf{I}$ denotes a $p \times p$ unit matrix. Variables $r, R, m$ and $M$, are the orders or the respective polynomials. Polynomials $\Phi_{t}(B)$ and $\Theta_{t}(B)$ respectively describe the so called regular $\mathrm{AR}$ and moving average (MA) factors, whereas $\boldsymbol{\Phi}_{t}^{(S)}(B)$ and $\boldsymbol{\Theta}_{t}^{(S)}(B)$ correspond to stochastic seasonal or periodic factors, where the period is denoted by $S$. Finally, $\mathbf{D}(B)$ and $\mathbf{D}^{(S)}(B)$ are diagonal matrix operators of size $p \times p$ that indicate regular and seasonal differencing:

$$
\begin{gathered}
\mathbf{D}(B)=\operatorname{diag}\left[\begin{array}{llll}
(1-B)^{d_{1}} & (1-B)^{d_{2}} & \ldots & (1-B)^{d_{p}}
\end{array}\right] \\
\mathbf{D}^{(S)}(B)=\operatorname{diag}\left[\begin{array}{llll}
\left(1-B^{S}\right)^{D_{1}} & \left(1-B^{S}\right)^{D_{2}} & \ldots & \left(1-B^{S}\right)^{D_{p}}
\end{array}\right]
\end{gathered}
$$

where $d_{1}, d_{2}, \ldots, d_{p}$, and $D_{1}, D_{2}, \ldots, D_{p}$ are respectively the orders of regular and seasonal differencing. Such regular and seasonal differencing is usually required for nonstationary or seasonal time series, respectively (Wei, 1993). It should be noted that the required order of differencing can generally be different in different channels. An important feature of the proposed vector ARIMA model is that the coefficients of polynomials $\boldsymbol{\Phi}_{t}(B), \boldsymbol{\Phi}_{t}^{(S)}(B)$, $\boldsymbol{\Theta}_{t}(B)$, and $\boldsymbol{\Theta}_{t}^{(S)}(B)$ are not assumed to be constant but are rather allowed to vary with time, which is highlighted by the subscript " $t$ ".

\subsection{Extended Kalman filter}

In this study, the coefficients of the vector seasonal ARIMA model are identified and unusual variations in their values are tracked as possible indicators of structural changes in the analyzed mechanical system. For the purpose of parameter identification we use the Kalman filter - a tool widely used for recursive identification. Due to the presence of the MA components in the considered model, a nonlinear state space realization arises, as 
demonstrated later, and the so called extended Kalman filter method is adopted. The derivation of the extended Kalman filter that follows is adopted after Harvey (1989).

Consider the following nonlinear state space model:

$$
\begin{gathered}
\mathbf{y}_{t}=\mathbf{z}_{t}\left(\boldsymbol{\alpha}_{t}\right)+\boldsymbol{\varepsilon}_{t} \\
\boldsymbol{\alpha}_{t}=\mathbf{t}_{t}\left(\boldsymbol{\alpha}_{t-1}\right)+\boldsymbol{\eta}_{t}
\end{gathered}
$$

Equation (9) is referred to as the measurement equation, and Equation (10) as the transition equation, respectively, where $\mathbf{y}_{t}$ is the vector of outputs and $\alpha_{t}$ is the vector of states. The elements of $\mathbf{z}_{t}\left(\alpha_{t}\right)$ and $\mathbf{t}_{t}\left(\alpha_{t-1}\right)$ are not necessarily linear functions of elements of the state vector. Vectors $\varepsilon_{t}$ and $\eta_{t}$ represent multivariate zero mean Gaussian disturbances with covariance matrices $\mathbf{H}_{t}$ and $\mathbf{Q}_{t}$, respectively. These disturbances can also be contemporaneously correlated, such that

$$
E\left(\boldsymbol{\eta}_{t} \boldsymbol{\varepsilon}_{s}^{\prime}\right)=\left\{\begin{array}{cc}
\mathbf{G}_{t}, & t=s \\
\mathbf{0}, & t \neq s
\end{array}\right.
$$

where $E$ denotes the expected value operator, and apostrophe denotes transposition.

The nonlinear functions $\mathbf{z}_{t}\left(\alpha_{t}\right)$ and $\mathbf{t}_{t}\left(\alpha_{t-1}\right)$ can be linearized through expansion in the Taylor series around conditional means of the state vector. The linearization yields:

$$
\begin{gathered}
\mathbf{z}_{t}\left(\boldsymbol{\alpha}_{t}\right)=\mathbf{z}_{t}\left(\mathbf{a}_{t \mid t-1}\right)+\left.\frac{\partial \mathbf{z}_{t}\left(\boldsymbol{\alpha}_{t}\right)}{\partial \boldsymbol{\alpha}_{t}^{\prime}}\right|_{\boldsymbol{\alpha}_{t}=\mathbf{a}_{t \mid l-1}}\left(\boldsymbol{\alpha}_{t}-\mathbf{a}_{t \mid t-1}\right) \\
\mathbf{t}_{t}\left(\boldsymbol{\alpha}_{t-1}\right)=\mathbf{t}_{t}\left(\mathbf{a}_{t-1}\right)+\left.\frac{\partial \mathbf{t}_{t}\left(\boldsymbol{\alpha}_{t-1}\right)}{\partial \boldsymbol{\alpha}_{t-1}^{\prime}}\right|_{\boldsymbol{\alpha}_{t-1}=\mathbf{a}_{t-1}}\left(\boldsymbol{\alpha}_{t-1}-\mathbf{a}_{t-1}\right)
\end{gathered}
$$

where vectors $\mathbf{a}_{t \mid t-1}$ and $\mathbf{a}_{t-1}$ are respectively the optimal estimator of the state vector $\alpha_{t}$ conditional on the information available at time $t$-1, and the optimal estimator of the state vector $\alpha_{t-1}$ conditional on the information available at time $t-1$.

Introducing the following notation: 


$$
\begin{aligned}
& \mathbf{Z}_{t}=\left.\frac{\partial \mathbf{z}_{t}\left(\boldsymbol{\alpha}_{t}\right)}{\partial \boldsymbol{\alpha}_{t}^{\prime}}\right|_{\boldsymbol{\alpha}_{t}=\mathbf{a}_{t t-1}} \\
& \mathbf{T}_{t}=\left.\frac{\partial \mathbf{t}_{t}\left(\boldsymbol{\alpha}_{t-1}\right)}{\partial \boldsymbol{\alpha}_{t-1}^{\prime}}\right|_{\boldsymbol{\alpha}_{t-1}=\mathbf{a}_{t-1}}
\end{aligned}
$$

the optimal estimation, $\mathbf{a}_{t}$, of the state vector $\alpha_{t}$ conditional on the information available at time $t$ can be obtained through recursive application of the following prediction equations:

$$
\begin{gathered}
\mathbf{a}_{t \mid t-1}=\mathbf{t}_{t}\left(\mathbf{a}_{t-1}\right) \\
\mathbf{P}_{t \mid t-1}=\mathbf{T}_{t} \mathbf{P}_{t-1} \mathbf{T}_{t}^{\prime}+\mathbf{Q}_{t}
\end{gathered}
$$

and updating equations:

$$
\begin{gathered}
\mathbf{a}_{t}=\mathbf{a}_{t \mid t-1}+\left(\mathbf{P}_{t \mid t-1} \mathbf{Z}_{t}^{\prime}+\mathbf{G}_{t}\right) \mathbf{F}_{t}^{-1}\left[\mathbf{y}_{t}-\mathbf{Z}_{t}\left(\mathbf{a}_{t \mid t-1}\right)\right] \\
\mathbf{P}_{t}=\mathbf{P}_{t \mid t-1}-\left(\mathbf{P}_{t \mid t-1} \mathbf{Z}_{t}^{\prime}+\mathbf{G}_{t}\right) \mathbf{F}_{t}^{-1}\left(\mathbf{Z}_{t} \mathbf{P}_{t \mid t-1}^{\prime}+\mathbf{G}_{t}^{\prime}\right)
\end{gathered}
$$

with

$$
\mathbf{F}_{t}=\mathbf{Z}_{t} \mathbf{P}_{t \mid t-1} \mathbf{Z}_{t}^{\prime}+\mathbf{Z}_{t} \mathbf{G}_{t}+\mathbf{G}_{t}^{\prime} \mathbf{Z}_{t}^{\prime}+\mathbf{H}_{t}
$$

Matrix $\mathbf{P}_{t}$ is the covariance matrix of the estimation error:

$$
\mathbf{P}_{t}=E\left[\left(\boldsymbol{\alpha}_{t-1}-\mathbf{a}_{t \mid t-1}\right)\left(\boldsymbol{\alpha}_{t-1}-\mathbf{a}_{t \mid t-1}\right)^{\prime}\right]
$$

Taken together, Equations (16)-(20) form the Kalman filter.

For the purpose of identification of coefficients of the vector ARIMA model of Equation (1), the output and state vectors were respectively defined as follows:

$$
\begin{aligned}
& \mathbf{y}_{t}=\mathbf{x}_{t}
\end{aligned}
$$

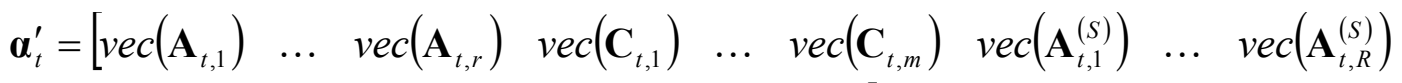

$$
\begin{aligned}
& \left.\operatorname{vec}\left(\mathbf{C}_{t, 1}^{(S)}\right) \quad \ldots \quad \operatorname{vec}\left(\mathbf{C}_{t, M}^{(S)}\right) \quad \mathbf{e}_{t}^{\prime} \quad \ldots \quad \mathbf{e}_{t-\max (m, S M)}^{\prime}\right]
\end{aligned}
$$

where the operator $\operatorname{vec}(\mathbf{V})$ reorganizes entries of matrix $\mathbf{V}$ into a row vector. Vector $\mathbf{x}_{t}$ denotes strains recorded at time $t$, and the entries of the state vector are coefficients of the vector 
ARIMA model and the noise time series defined in Equations (1) and (3)-(6). With the above definitions, the measurement equation (Equation 9) clearly includes multiplicative terms in the entries of the state vector, resulting from term such as $\mathbf{C}_{t, 1} \mathbf{e}_{t-i}$ and $\mathbf{C}_{t, 1}^{(S)} \mathbf{e}_{t-S i}$, and is therefore nonlinear. Note also, that with the state vector defined as in Equation (23) there is no output noise, i.e. $\boldsymbol{\varepsilon}_{t}=\mathbf{0}$.

The transition equation (Equation 10) was assumed in the following linear form:

$$
\boldsymbol{\alpha}_{t}=\boldsymbol{\alpha}_{t-1}+\boldsymbol{\eta}_{t}
$$

This type of time series is often referred to as the random walk model (Wei, 1993). The rationale behind this choice is that under the null hypothesis the parameters of the system are assumed not to change with time, except for some stochastic uncertainty in their identification.

\subsection{Outlier detection and intervention analysis}

It is desirable to have an analytical tool which could be used to pick up and quantify unusual events and changes, such as level shifts, in a time series. The problem may be addressed by outlier detection and intervention analysis. Outlier detection techniques examine a time series in order to determine whether a particular observation protrudes unduly from the regimen of the analyzed time series. Intervention analysis is a next logical step after outlier detection. Intervention analysis enables examination of what happened to the time series after an unusual event and checking if this event had a permanent impact or only a transient one. In what follows, we describe an integrated approach to outlier detection and intervention analysis presented by Pankratz (1991).

Consider the following univariate AR model with an exogenous input: 


$$
x_{t}=\frac{\omega(B)}{(1-B)^{d} \delta(B)} I_{t}^{T}+\frac{1}{\phi(B)} e_{t}
$$

where $\left\{x_{t}\right\}$ is the analyzed univariate time series, and $\left\{e_{t}\right\}$ is a zero mean Gaussian white noise. $\phi(B)$ is a AR polynomial in the backshift operator $B$, having a general form similar to those of Equation (3), except being a univariate and not matrix polynomial. This polynomial describes the behavior of the time series at hand when it is not disturbed by any external inputs except the noise $\left\{e_{t}\right\} . I^{T}$ is, on the other hand, the exogenous impulse time series defined as

$$
I_{t}^{T}= \begin{cases}1, & t=T \\ 0, & t \neq T\end{cases}
$$

Polynomials $\omega(B)$ and $\delta(B)$ describe the effect of an exogenous impulse input. They may generally have forms similar to Equation (3); in this study, however, their forms are restricted to the following simple cases:

$$
\begin{gathered}
\omega(B)=\omega_{0} \\
\delta(B)=1-\delta_{1} B
\end{gathered}
$$

where $\omega_{0}$ quantifies the magnitude of the exogenous input effect. The parameter $\delta_{1}$ is confined in the interval $0 \leq \delta_{1}<1$. Depending on the value of $\delta_{1}$ and the order of differencing operator $d$ in Equation (25), the development of the input effect in time can have several forms listed in Table 1 and schematically depicted in Figure 3. One may then observe a transient change (Fig. 3a), a level shift (Fig. 3b), a gradual change (Fig 3c), and a linear trend (Fig. 3d).

Pankratz (1991) describes an integrated method for outlier detection and estimation of response magnitude $\omega_{0}$ when the polynomials $\phi(\mathrm{B})$ and $\delta(\mathrm{B})$ in Equation (25) and the 
variance of noise $\left\{e_{t}\right\}$, denoted by $\sigma_{e}^{2}$, are known. Defining the following two auxiliary time series:

$$
W_{t}=\frac{\phi_{t}(B)}{(1-B)^{d} \delta(B)} I_{t}^{T}
$$

and

$$
\xi_{t}=\omega_{0} W_{t}+e_{t}
$$

the least square estimator of the response magnitude at time $T$, denoted as $\omega_{0, T}$, is

$$
\omega_{0, T}=\frac{\sum_{t} W_{t} \xi_{t}}{\sum_{t} W_{t}^{2}}
$$

Under the null hypothesis that there are no outliers in the examined time series, this estimator can be shown to be normally distributed with a zero mean and variance $\sigma_{\omega}^{2}$ equal to

$$
\sigma_{\omega}^{2}=\frac{\sigma_{e}^{2}}{\sum_{t} W_{t}^{2}}
$$

Based on the above statistical results regarding the distribution of $\omega_{0, T}$, an observation is declared to be an outlier if its absolute value normalized by the standard deviation exceeds a preset threshold $C$ :

$$
\frac{\left|\omega_{0, T}\right|}{\sigma_{\omega}}>C
$$

The knowledge of statistical distribution of $\omega_{0, T}$ enables relating the threshold's value to the normal distribution, and significance levels could then be assigned to the decision whether a tested observation is an inlier or an outlier.

In practice, however, polynomial $\delta(\mathrm{B})$ and the order of differencing $d$ are usually unknown a priori and have to be assumed. One has typically to test several possible values of 
$\delta_{1}$ and choose that offering the best fit to the observed time series. The order of differencing, $d=0$ or 1 , also needs to be determined. These decisions can be supported statistically by choosing the values of $\delta_{1}$ and $d$ for which the quotient of Equation (32) assumes the highest value.

\section{APPLICATION}

\subsection{Analysis of single signal recorded during bridge construction}

The studied bridge was built using the balanced cantilever method and in-situ concreting (Mathivat, 1983). The construction process of the girder started from a pier, and comprised repetitive steps during which two segments of the girder beam, symmetrically located with respect to the pier, were first concreted, then stressed using tendons, and finally concreting form traveler was shifted in preparation for casting of another segment. At the end of construction of the span, the closure segment was cast and continuity or integration tendons were stressed, thus changing the static system of the bridge from separate cantilevers into a continuous beam. Table 2 lists major stages of the construction schedule together with the times when they were carried out. Each of these events, i.e. concreting, tensioning, and shifting of form traveler lasted for a few hours and were separated by a few days' time intervals. They were performed according to a construction schedule and well documented. At the same time, these events can be expected to produce significant, sudden changes in strains, and possibly also changes in the mechanical system of the bridge. Thus, the concreting, tensioning and form traveler shifting events can be used to shed some light onto the efficiency and performance of the proposed analytical method for monitoring the state of the structure. For example, the cable tensioning events are likely to produce similar effects as possible cable failures during the service life of the bridge. It is interesting to examine 
whether the method is capable of identifying significant construction events, and also to see to what extent such events are different from other strain changes caused, e.g., by abnormal loading or temperature.

This section of the paper is devoted to examining the performance of the proposed analytical method using signals recorded during construction and shortly after opening of the bridge to the traffic by the strain gauge located in Segment 31, close to the top of the girder (see Fig. 2), which is denoted by SG31-1. The strain time series starts on April 29, 1997 at 00:00 hours and comprises 2944 hourly sampled measurements. This time series is shown in Figure 4. The sign convention is such that positive strains denote contraction; in addition, the numerical values of strains, given here in the microstrain units $\left(1 \mu \varepsilon=10^{-6}\right)$, correspond to relative rather than absolute strains and are measured from unknown reference levels, which are generally different for different channels.

Preliminary inspection showed that the analyzed strain time series is nonstationary and in addition, as could be expected, has a clear 24-hour seasonal component due to the daily temperature cycle. Hence, the original signal was first differenced using operators $(1-B)$ and $\left(1-B^{24}\right)$ once each. By trying several orders for the AR and MA regular and seasonal polynomials, it was finally decided that the model orders $r=1, R=0, m=0$ and $M=1$ would provide sufficient accuracy. This conclusion was reached by observing the autocorrelation function of the residual noise $\left\{\mathbf{e}_{t}\right\}$ [see Eq. (1)], which did not show significant values for the chosen orders, i.e. $\left\{\mathbf{e}_{t}\right\}$ became a white noise. Results of the identification using the extended Kalman filter method are shown in Figures 5 and 6 . The first regular AR coefficient, $\mathbf{A}_{t, 1}$, is shown in Figure 5, and the first seasonal MA coefficient, $\mathbf{C}_{t, 1}^{(S)}$, in Figure 6, respectively. It can be seen in these figures that the identified values of the coefficients exhibit quite large variations, and notably many level shifts. 
In order to quantify the changes in the values of the ARIMA model coefficients, the statistical procedure laid out in Section 3.3. is used. Because the initial assumption or null hypothesis is that the values of each coefficient follow the random walk model of Equation (24), the AR polynomial $\phi(\mathrm{B})$ of Equation (25) becomes simply 1-B. As a preliminary visual inspection of plots of all coefficient time series, such as Figures 5 and 6 , showed that the level shift is the most frequent and obvious type of change, we first attempted to identify and quantify such step changes. According to Table 1 , a level shift corresponds to $d=1$ and $\delta_{1}=0$. In this case, the following time series take the simple forms: $W_{t}=I_{t}^{T}$ (impulse), and $\omega_{0, t}=\xi_{t}=e_{t}=(1-B) x_{t}$. The estimated values of the magnitudes of level shifts, $\omega_{0, t}$, for the first regular AR coefficient $\mathbf{A}_{t, 1}$ are shown in Figure 7. Several large spikes can be seen in this time series indicating possible outliers. If we were to proceed with the proposed rigorous procedure we would now have to estimate the noise variance $\sigma_{e}^{2}$ and decide on a confidence level with which we want to identify outliers. This can be done easily if a sufficiently large data set is given which contains no or only a small number of outliers. The time series of $\omega_{0, t}$ has, however, many spikes and large excursions from its mean value, and it is difficult to isolate with confidence a long portion of the series without outliers. Faced with such an obstacle, we adopted a pragmatic approach and decided to focus our attention on cable tensioning events listed in Table 2. This approach may be supported by the following rationale. Firstly, it turns out that some of the tensioning events result in the largest changes in the values of ARIMA coefficients. Secondly, for this post-tensioned bridge, the integrity of the cables is by far the most critical factor determining its structural health. The values of the threshold $C$ were then chosen independently for each ARIMA coefficient so as to produce approximately $1.5 \%$ of outliers. Such values guarantee that the number of excursions beyond the threshold is small and so excessive numbers of alarms can be avoided, but at the same 
time all the tensioning events are detected along with other significant changes in the strain time series.

A total of 46 events were identified among the values of the ARIMA coefficients through outlier analysis. It is important to mention that in this discussion, we refer to a single observation that exceeds the threshold value as an "outlier". An "event", on the other hand, is typically related to some abnormal changes in the strain time series that lasted for a few hours. An event often causes several values of the ARIMA coefficients to exceed the threshold value. Thus, several subsequent or very closely separated outliers are grouped and identify a single event. Figure 8 zooms into the time series of strains and coefficient $\mathbf{A}_{t, 1}$ for a tensioning event T24 and another identified but unknown construction event. It can be seen that for these two exemplary events sharp changes in strains are present and they correspond to step changes in the values of the AR coefficient. Thus, it can be concluded that cable tensioning and other events do change the mechanical system as modeled by the ARIMA model and that they can be detected by observing variations in the ARIMA model. Table 3 shows a summary of identification of all tensioning events. While all 5 tensioning events were detected in the time series of the first regular AR coefficient, $\mathbf{A}_{t, 1}$, the first seasonal MA coefficient, $\mathbf{C}_{t, 1}^{(S)}$, missed 2 of them. Thus, $\mathbf{A}_{t, 1}$ appears to be a good candidate signal feature to detect tensioning events, but $\mathbf{C}_{t, 1}^{(S)}$ does not seem to be a reliable observation for such a type of events.

4.2 Analysis of multivariate signal recorded after bridge construction

In the previous section, a strain signal from a single strain gauge was analyzed. The proposed theoretical framework is, however, capable of examining multivariate time series, too. Such as analysis is undertaken in this section. The main objective is to assess whether 
new significant information about the behavior of the structure can be obtained via application of a vector ARIMA model.

For the purpose of analysis of a multivariate signal, a portion of strain time series recorded by two strain gauges during post-construction stage was chosen. The first gauge is SG31-1, used also in the previous analysis, the second one is SG23-1, located in Segment 23 (one fourth of the span length) close to the top of the beam, similarly to SG31-1 (see Fig. 2). The record starts on July 8, 1999 at 17:00 hours and comprises 1264 hourly sampled measurements. These two time series are shown in Figure 9.

By extending an analysis from a univariate case to a multivariate or vector model one expects to discover new information about the studied system and/or explain some of its behaviors that the univariate model cannot describe. In the context of the proposed monitoring method it is important to assess whether a vector ARIMA model, which accounts for correlations among signals recorded at various locations in the bridge structure, performs better than a set of univariate ARIMA models, formulated independently for each data channel. To obtain some insight into this problem we analyzed the post-construction twochannel data twice. The first analysis used two independent ARIMA models for each strain gauge's time series. The second analysis identified matrix coefficients of a vector ARIMA model for the two strain time series analyzed jointly.

The orders of ARIMA models were in this case assumed as $r=4, R=0, m=0$ and $M=1$, because it turned out that as many as 4 regular AR terms are necessary for the residual time series $\left\{\mathbf{e}_{t}\right\}$ [see Eq. (1)] to become a white noise. The values of the threshold $C$ were chosen independently for each ARIMA coefficient so as to produce approximately $1 \%$ of outliers. All available model coefficients were used in the simulations. It should be noted that vector models have also off-diagonal coefficients, thus the total number of coefficients increases for such models. A total of 16 events were identified jointly by the two univariate models, 
whereas the vector model singled out 25 unusual events. There was only one event identified by the univariate models that was not spotted by the vector model; the reverse was true for 10 incidents. While these observations may suggest that a vector approach could be more reliable, any conclusions must be made very cautiously. By including and retaining more coefficients in the model a chance of identifying outlying observations increases, even if these observation are just false alarms. Clearly, a more rigorous statistical methodology could help to resolve this dilemma. However, as previously mentioned, the number of outlying observations in the time series of ARIMA coefficients is large, making any statistical analysis very difficult.

An example of an identified post-construction event is shown in Figure 10. Figures 10a) and 10b) zoom into the strain time series associated with the event and recorded by gauges SG31-1 and SG23-1, respectively, while Figures 10c) and 10d) show values of the selected matrix ARIMA coefficients $\mathbf{A}_{t, 1}(1,1)$ and $\mathbf{A}_{t, 1}(1,2)$, respectively. It can be seen that, similarly to the monitoring during construction, level shifts in the ARIMA coefficient values correspond to significant abrupt changes in the strain signals. This supports the expectation that the method can be used for monitoring of structural health. It is also interesting to note that the level shift can be noticed in both diagonal and off-diagonal matrix ARIMA coefficients. Thus, the analyzed events appear to alter not only the correlations between the current observation and its predecessors from the same data channel, but also the correlations between observations from spatially separated sensors.

\section{CONCLUSIONS}

This study proposed and examined the application of concepts of time series analysis to process data from a continuously operating SHM system installed in a major bridge structure. The recorded static strain data were modeled using ARIMA models. Two types of models 
were used. The first one was a univariate model that described the signal recorded by a single strain sensor. The other model was a multivariate model, which enabled simultaneous analysis of signals from multiple channels and took into account the correlation among the signals. The coefficients of the ARIMA models were allowed to vary with time. They were identified on-line using the extended Kalman filter, and chosen as structural damage or change sensitive features. The method was first applied to strains recorded during bridge construction, when structural changes corresponded to the known significant events such as cable tensioning. This allowed some form of verification of the method's ability to detect changes in the structural behavior. The proposed method passed this basic test and was able to detect changes caused by tensioning of tendons. In the subsequent investigations, the method was used to analyze signals recorded during post-construction period when the bridge was in service. During that period some significant changes in strain time series and associated ARIMA models were also revealed, showing that the method can provide information on structural performance under usual environmental and operational conditions.

While this investigation shows that the studied concepts from time series analysis could be useful in structural monitoring, there are several important questions that need more detailed and careful evaluation. For example, the proposed method does not make use of all available measured data, notably temperature. It is possible that some of the observed model changes may in fact be caused by abnormal temperature variations and are not associated with structural changes. To clarify this, a modified, augmented ARIMAX (ARIMA with exogenous inputs) model, which includes available temperature measurements, will be studied. This model will use multiple inputs to account for the influence of both spatial average of temperatures as well as spatial temperature differentials across the girder.

More importantly, however, the proposed method enables detection of unusual events in the strain time series and therefore signalizes a possible onset of structural change or 
damage, but it does not decisively tell whether any damage has actually been sustained by the structure. Neither does it provide any characteristics of the damage, such as its nature, severity or location. For practical applications, these limitations need to be overcome so that infrastructure managers can benefit from the method and use it confidently and effectively. Linking the observed patterns of changes in ARIMA models with structural changes could be achieved using analytical, physics-based structural models and numerical simulations of different damage scenarios, such as post-tensioning tendon failure or foundation settlement. Ongoing investigations aim at improving the discussed methodology along the above suggestions. 


\section{REFERENCES}

Aktan, A. E., Catbas, F. N., Grimmelsman, K., Pervizpour, M., Curtis, J., Shen, K., Qin, X. \& Cinar, O. A. (2002), Health monitoring for effective management of infrastructure, in Balageas, D. L., Ed., Structural Health Monitoring 2002, Destech Publications, Lancaster, 1245-1253.

Alampalli, S. \& Fu, G. (1994), Instrumentation for remote and continuous monitoring of structure conditions, Transportation Research Record, 1432, 59-67.

Bakht, B. \& Jaeger, L. G. (1990), Bridge testing: a surprise every time, Journal of Structural Engineering, ASCE, 116(5), 1370-1383.

Cornwell, P., Farrar, C. R., Doebling, S. W. \& Sohn, H. (1999), Environmental variability of modal properties, Experimental Techniques, 23(6), 45-48.

Farrar, C. R., Cornwell, P. J., Doebling, S. W. \& Prime, M. B. (2000). Structural Health Monitoring Studies of the Alamosa Canyon and I-40 Bridges, Los Alamos National Laboratory, Los Alamos.

Heywood, R. J., Roberts, W., Taylor, R. \& Anderson, R. (2000), Fitness-for-purpose evaluation of bridges using health monitoring technology, Transportation Research Record, 1696, 193-201.

Harvey, A. C. (1989), Forecasting, structural time series models and the Kalman filter, Cambridge University Press, New York.

Kullaa, J. (2002), Elimination of environmental influences from damage-sensitive features in a structural health monitoring system, in Balageas, D. L., Ed., Proceedings of the 1st European Workshop on Structural Health Monitoring, Destech Publications, Lancaster, 742-749.

Mahmoud, M., Abe, M. \& Fujino, Y. (2001), Analysis of suspension bridge by ambient vibration measurement using the time domain method and its application to health 
monitoring, in Balageas, D. L., Ed., Proceedings of the 19th International Modal Analysis Conference, 504-510.

Mathivat, J. (1983), The cantilever construction of prestressed concrete bridges. John Wiley \& Sons, New York.

Moyo, P. \& Brownjohn, J. M. W. (2002a), Detection of anomalous structural behavior using wavelet analysis, Mechanical Systems and Signal Processing, 16(2/3), 429-445.

Moyo, P. \& Brownjohn, J. M. W. (2002b), Application of Box-Jenkins models for assessing the effect of unusual events recorded by structural health monitoring systems, Structural Health Monitoring, 1(2), 149-160.

Omenzetter, P., Brownjohn J., M., W. \& Moyo, P. (2004), Identification of unusual events in multi-channel bridge monitoring data. Mechanical Systems and Signal Processing, 18, 409-430.

Pankratz, A. (1991), Forecasting with dynamic regression models. John Willey \& Sons, New York.

Phares, B. M., Rolander, D. D., Graybeal, B. A. \& Washer, G. A. (2001), Reliability of visual bridge inspection, Public Roads, 64(5), 22-29.

Salane, H. J., Baldwin, J. W. \& Duffield, R. C. (1981), Dynamics approach for monitoring highway bridge deterioration, Transportation Research Record, 832, 21-28.

Sohn, H., Dzwonczyk, M., Straser, E. G., Kiremidjian, A. S., Law, K. H., \& Meng, T. (1999), An experimental study of temperature effect on modal parameters of the Alamosa Canyon Bridge, Earthquake Engineering and Structural Dynamics, 28, 879-897.

Sohn, H., Czarnecki, J. A. \& Farrar, C. R. (2000), Structural health monitoring using statistical process control, Journal of Structural Engineering, ASCE, 126(11), 13561363. 
Sohn, H., Farrar, C. R., Hunter, N. \& Worden, K. (2001), Applying the LANL statistical pattern recognition paradigm for structural health monitoring to data from a surfaceeffect fast patrol boat, Los Alamos National Laboratory, Los Alamos.

Wei, W. W. S. (1993), Time Series Analysis. Addison-Wesley, Redwood City.

Wood, M. G. (1992), Damage analysis of bridge structures using vibrational techniques, Ph.D. thesis, University of Aston, Birmingham, UK. 


\section{Table titles and figure captions}

Table 1. Types of response due to impulse input.

Table 2. Bridge construction schedule.

Table 3. Results of detection of cable tensioning events.

Figure 1. The Singapore-Malaysia Second Link.

Figure 2. Locations of strain gauges at segment 31.

Figure 3. Pictograms of various types of response listed in Table 1: a) transient hange, b) level shift, c) gradual change, and d) linear trend.

Figure 4. Strains recorded at gauge SG31-1 during construction and shortly after opening.

Figure 5. Identified values of first regular AR polynomial coefficient, $\mathbf{A}_{t, 1}$, during bridge construction.

Figure 6. Identified values of first seasonal MA polynomial coefficient, $\mathbf{C}_{t, 1}^{(S)}$, during bridge construction.

Figure 7. Estimated values of level shifts $\omega_{0, t}$ for the first regular AR coefficient $\mathbf{A}_{t, 1}$.

Figure 8. Time series of strains and values of coefficient $\mathbf{A}_{t, 1}$ : a) strains corresponding to tensioning event T24, b) values of coefficient $\mathbf{A}_{t, 1}$ corresponding to tensioning event $\mathrm{T} 24, \mathrm{c})$ strains corresponding to unknown construction event, and d) values of coefficient $\mathbf{A}_{t, 1}$ corresponding to unknown construction event.

Figure 9. Strains recorded after construction: a) gauge SG31-1, and b) gauge SG23-1.

Figure 10. Time series of strains and values of ARIMA coefficients for a postconstruction event: a) strains recorded by SG31-1, b) strains recorded by SG23-1, c) values of coefficient $\mathbf{A}_{t, 1}(1,1)$ d) values of coefficient $\mathbf{A}_{t, 1}(1,2)$. 
Table 1. Types of response due to impulse input.

\begin{tabular}{|c|c|c|c|}
\hline \multirow{2}{*}{$\begin{array}{c}\text { Response } \\
\text { name }\end{array}$} & \multicolumn{2}{|c|}{ Parameter values } & \multirow{2}{*}{$\begin{array}{c}\text { Reference } \\
\text { figure }\end{array}$} \\
\cline { 2 - 3 } & $\mathrm{d}$ & $\delta_{1}$ & Figure $3 \mathrm{a}$ \\
\hline Transient change & $\mathrm{d}=0$ & $0 \leq \delta_{1}<1$ & Figure $3 \mathrm{~b}$ \\
\hline Level shift & $\mathrm{d}=1$ & $\delta_{1}=0$ & Figure 3c \\
\hline Gradual change & $\mathrm{d}=1$ & $0 \leq \delta_{1}<1$ & Figure $3 \mathrm{~d}$ \\
\hline Linear trend & $\mathrm{d}=2$ & $\delta_{1}=0$ & \\
\hline
\end{tabular}


Table 2. Bridge construction schedule.

\begin{tabular}{|l|c|c|}
\hline \multicolumn{1}{|c|}{ Activity } & Abbreviation & Time \\
\hline Concreting of Segment 27 & C27 & $91-95$ \\
\hline Tensioning of Segment 27 & T27 & $154-156$ \\
\hline Shifting of form off Segment 27 & F27 & $180-181$ \\
\hline Concreting of Segment 26 & C26 & $285-289$ \\
\hline Tensioning of Segment 26 & T26 & $326-330$ \\
\hline Shifting of form off Segment 26 & F26 & $350-355$ \\
\hline Concreting of Segment 25 & C25 & $499-502$ \\
\hline Tensioning of Segment 25 & T25 & $539-541$ \\
\hline Shifting of form off Segment 25 & F25 & $572-576$ \\
\hline Concreting of Segment 24 & C24 & $717-723$ \\
\hline Tensioning of Segment 24 & T24 & $779-781$ \\
\hline Shifting of form off Segment 24 & F24 & $950-953$ \\
\hline Concreting of Segment 23 & C23 & $1234-1240$ \\
\hline Tensioning of continuity tendons & TC & $1359-1363$ \\
\hline
\end{tabular}


Table 3. Results of detection of cable tensioning events.

\begin{tabular}{|c|c|c|}
\hline \multirow{2}{*}{ Event } & \multicolumn{2}{|c|}{ Detection results } \\
\cline { 2 - 3 } & $\mathbf{A}_{t, 1}$ & $\mathbf{C}_{t, 1}^{(S)}$ \\
\hline $\mathrm{T} 27$ & + & - \\
\hline $\mathrm{T} 26$ & + & + \\
\hline $\mathrm{T} 25$ & + & - \\
\hline $\mathrm{T} 24$ & + & + \\
\hline $\mathrm{TC}$ & + & + \\
\hline
\end{tabular}




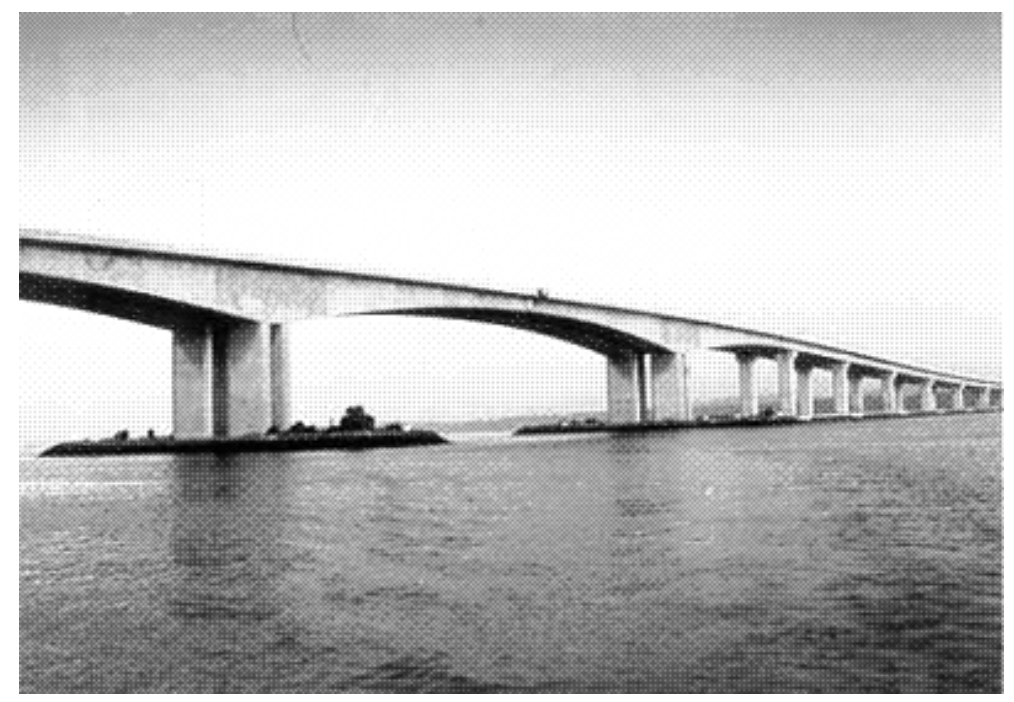

Figure 1. The Singapore-Malaysia Second Link. 


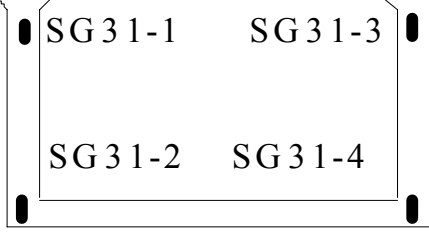

Figure 2. Locations of strain gauges at segment 31. 

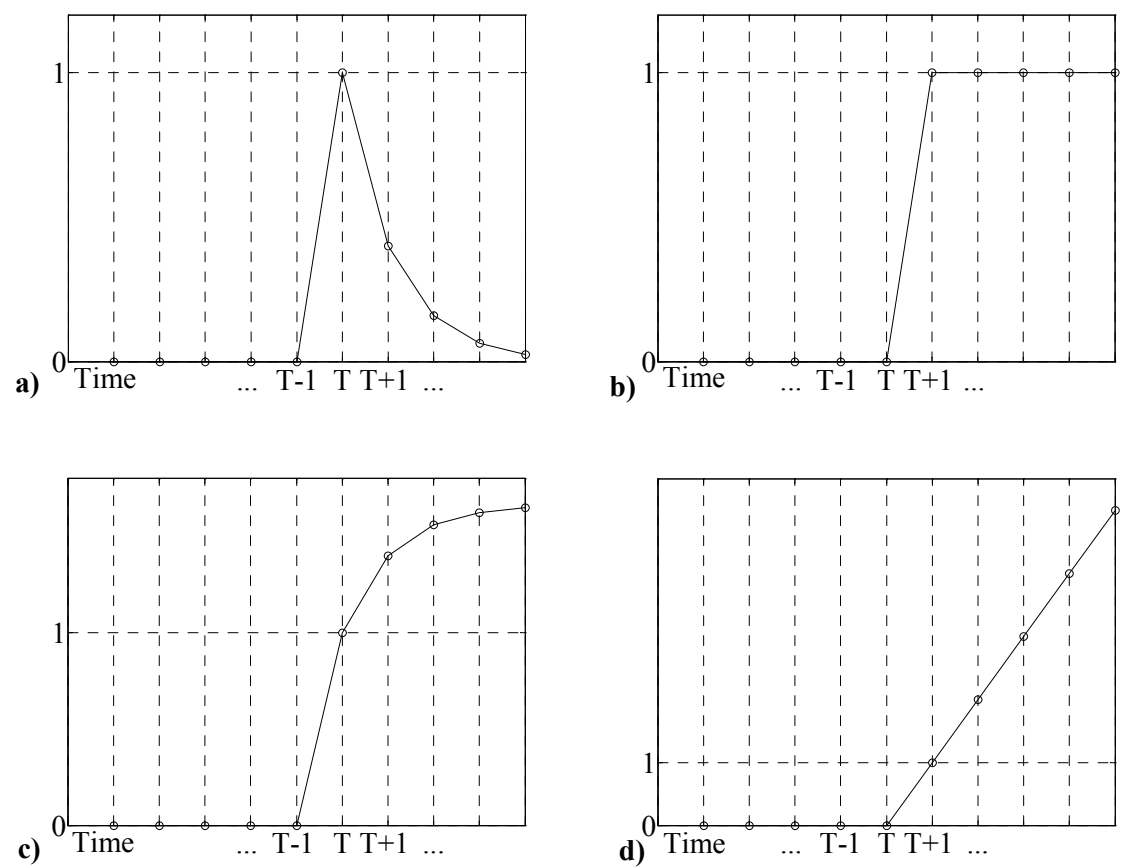

Figure 3. Pictograms of various types of response listed in Table 1: a) transient change, b) level shift, c) gradual change, and d) linear trend. 


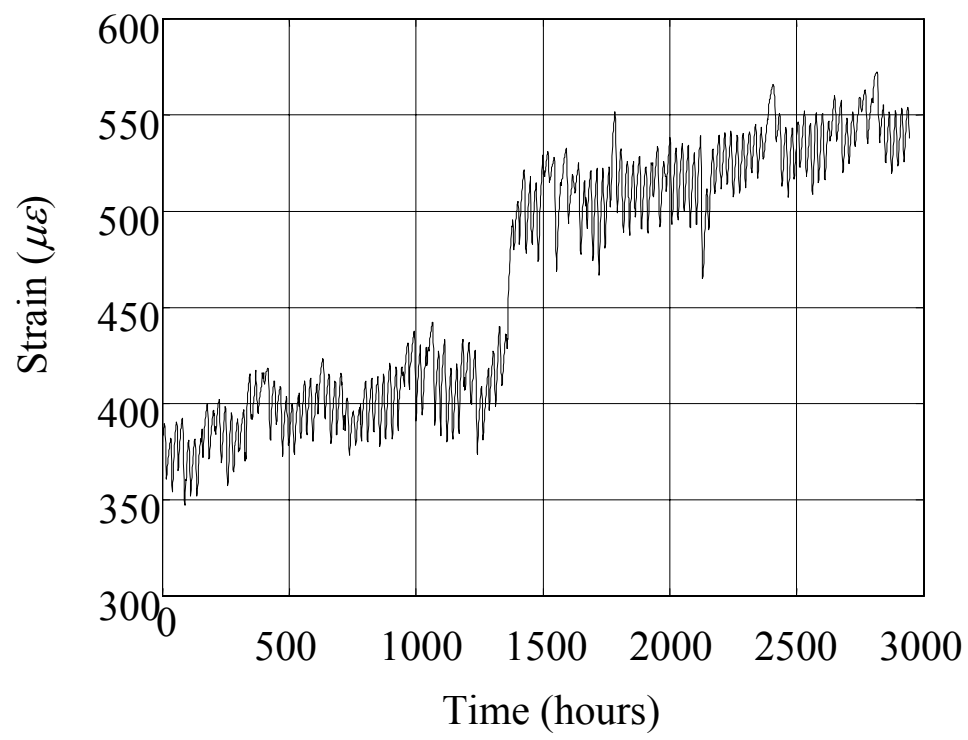

Figure 4. Strains recorded at gauge SG31-1 during construction and shortly after opening. 


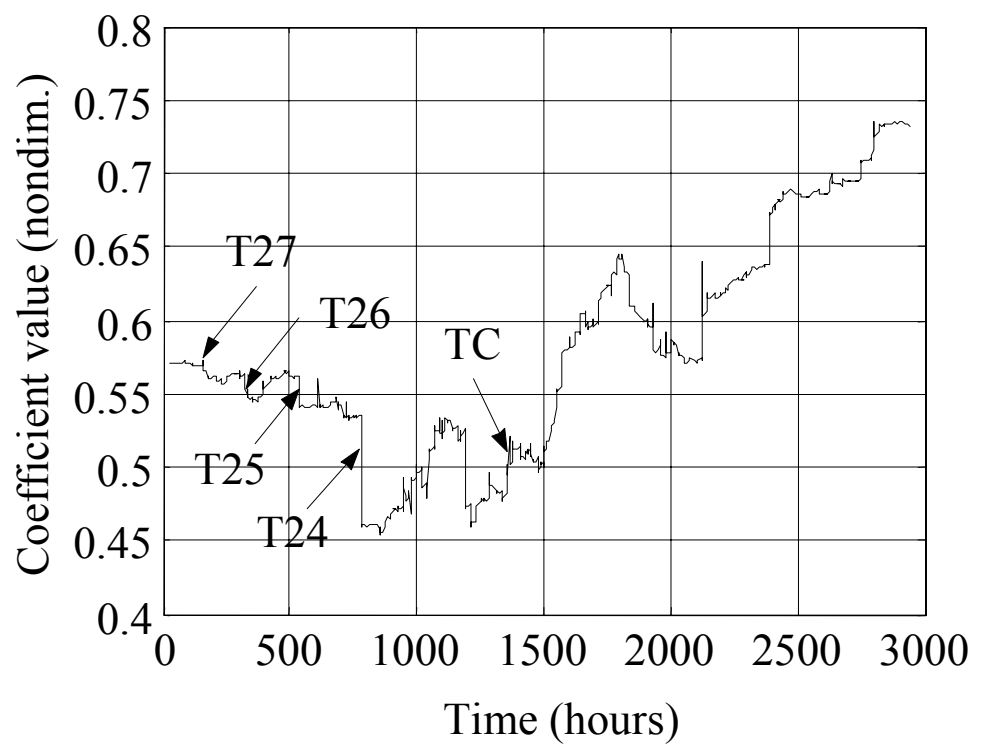

Figure 5. Identified values of first regular AR polynomial coefficient, $\mathbf{A}_{t, 1}$, during bridge construction. 


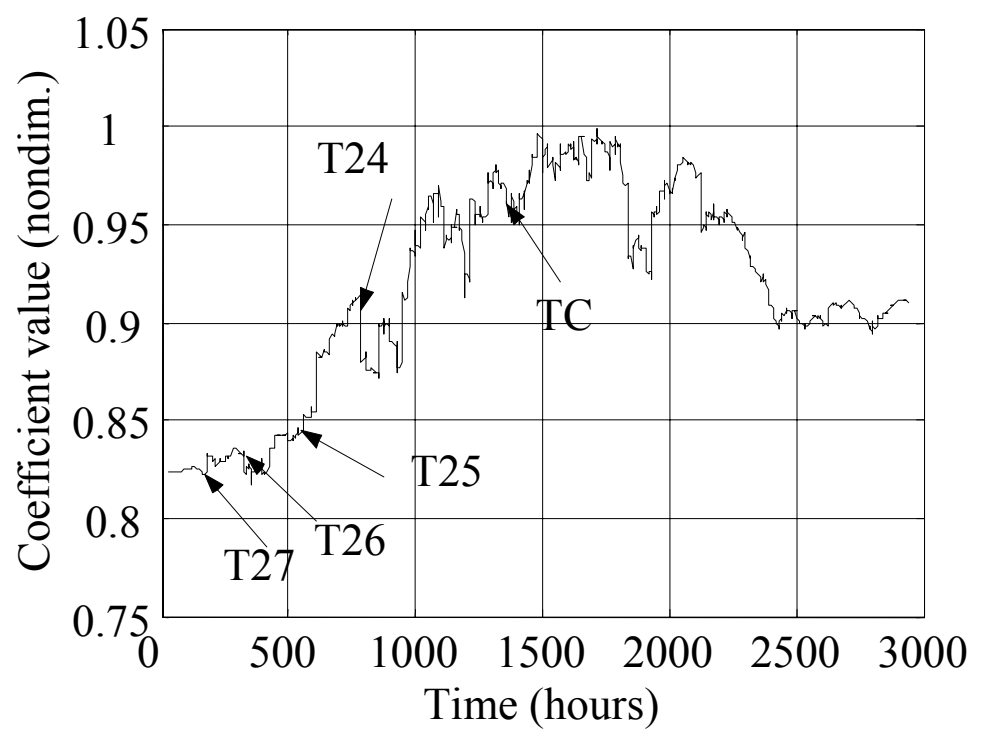

Figure 6. Identified values of first seasonal MA polynomial coefficient, $\mathbf{C}_{t, 1}^{(S)}$, during bridge construction. 


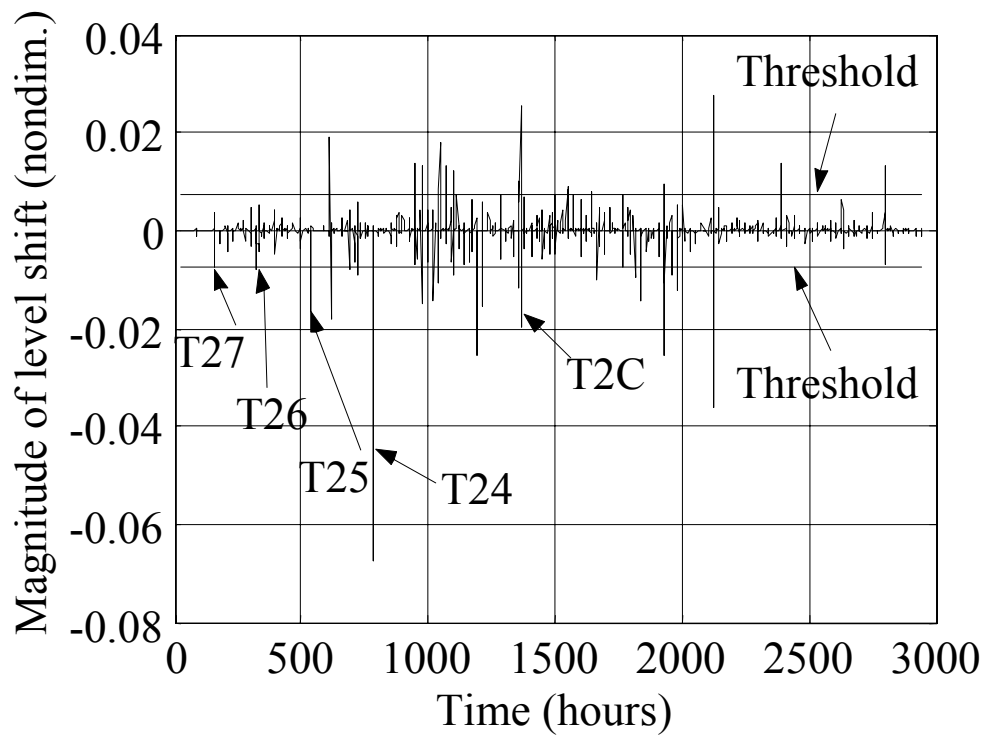

Figure 7. Estimated values of level shifts $\omega_{0, t}$ for the first regular AR coefficient $\mathbf{A}_{t, 1}$. 


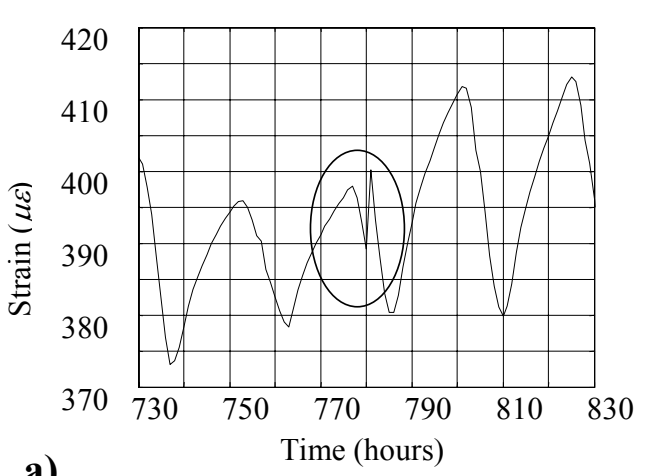

a)

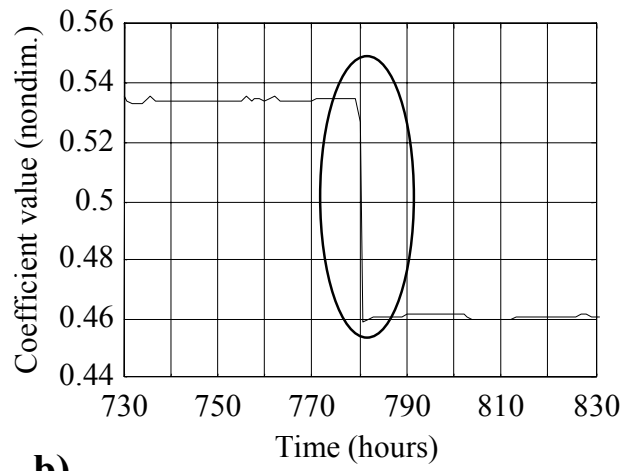

b)
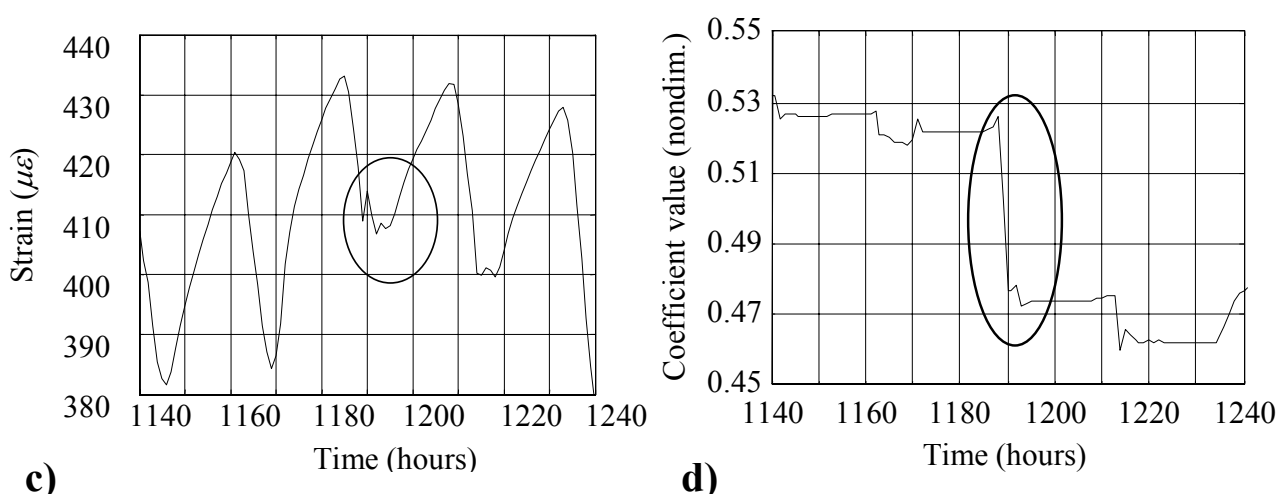

Figure 8. Time series of strains and values of coefficient $\mathbf{A}_{t, 1}$ : a) strains corresponding to tensioning event $\mathrm{T} 24, \mathrm{~b}$ ) values of coefficient $\mathbf{A}_{t, 1}$ corresponding to tensioning event $\mathrm{T} 24, \mathrm{c}$ ) strains corresponding to unknown construction event, and d) values of coefficient $\mathbf{A}_{t, 1}$ corresponding to unknown construction event. 


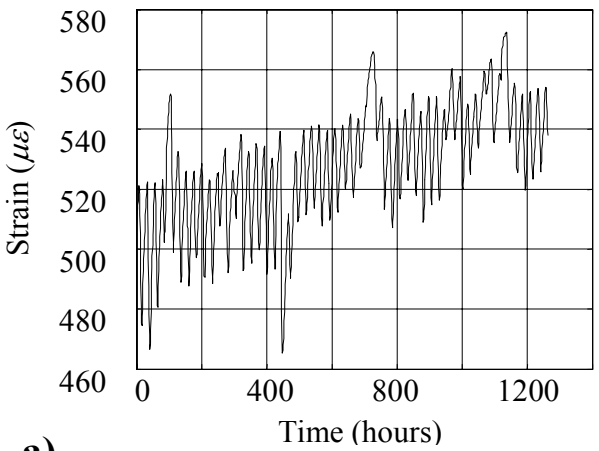

a)

Time (hours)

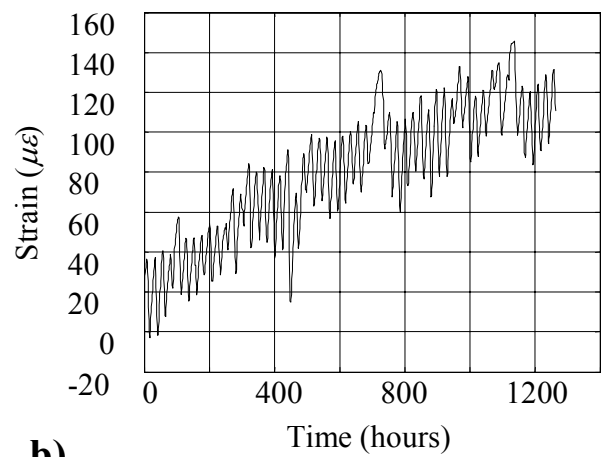

b)

Figure 9. Strains recorded after construction: a) gauge SG31-1, and b) gauge SG23-1. 


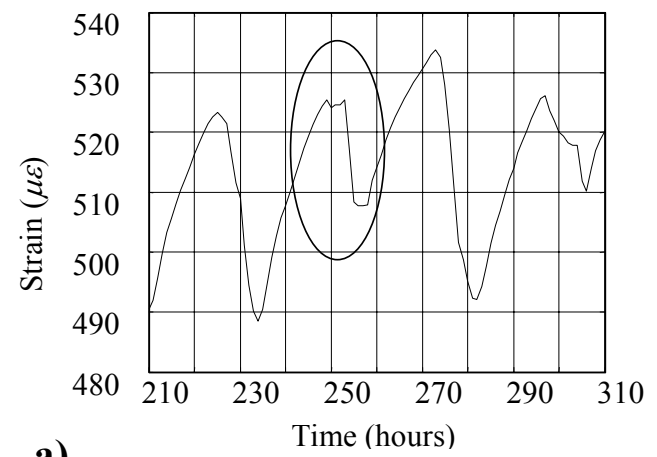

a)

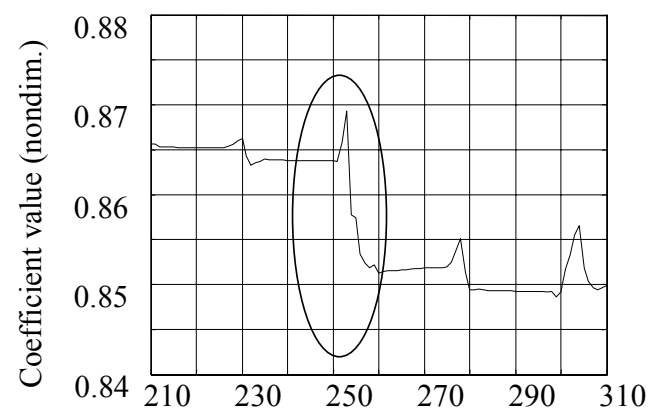

c)

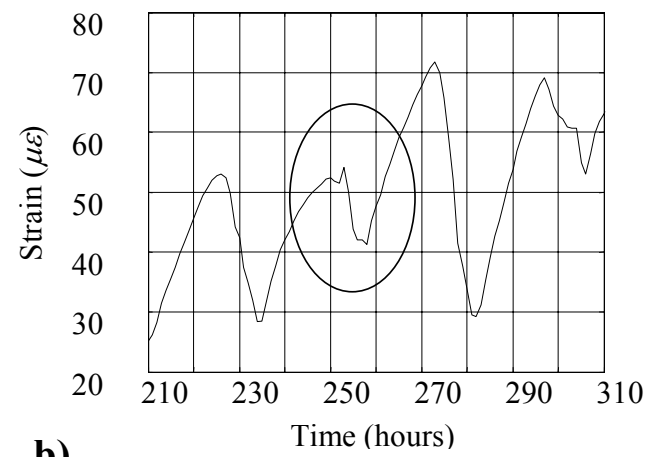

b)

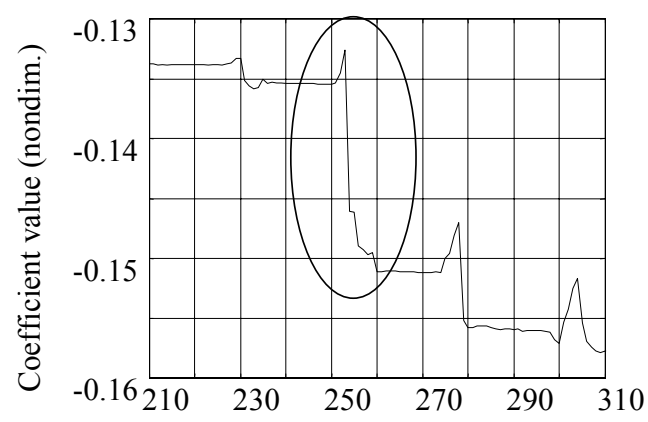

d)

Time (hours)

Figure 10. Time series of strains and values of ARIMA coefficients for a postconstruction event: a) strains recorded by SG31-1, b) strains recorded by SG23-1, c) values of coefficient $\mathbf{A}_{t, 1}(1,1)$ d) values of coefficient $\mathbf{A}_{t, 1}(1,2)$. 